\title{
Asymptotic freedom from the two-loop term of the $\beta$ function in a cubic theory
}

\author{
J. A. Gracey॰ \\ Theoretical Physics Division, Department of Mathematical Sciences, University of Liverpool, \\ P.O. Box 147, Liverpool, L69 3BX, United Kingdom
}

(Received 8 May 2020; accepted 12 June 2020; published 29 June 2020)

\begin{abstract}
We renormalize a six-dimensional cubic theory to four loops in the modified minimal subtraction $(\overline{\mathrm{MS}})$ scheme where the scalar is in a bi-adjoint representation. The underlying model was originally derived in a problem relating to gravity being a double copy of Yang-Mills theory. As a field theory in its own right we find that it has a curious property in that while unexpectedly there is no one-loop contribution to the $\beta$ function the two-loop coefficient is negative. It therefore represents an example where asymptotic freedom is determined by the two-loop term of the $\beta$ function. We also examine a multi-adjoint cubic theory in order to see whether this is a more universal property of these models.
\end{abstract}

DOI: $10.1103 /$ PhysRevD.101.125022

\section{INTRODUCTION}

Scalar $\phi^{3}$ theory in six dimensions has proved to be a useful laboratory or tool to explore major ideas in quantum field theory. For instance, after the discovery of asymptotic freedom in quantum chromodynamics (QCD) [1,2], it was used as a testing ground to study the implications of this property. This was because it was shown that sixdimensional $\phi^{3}$ theory was renormalizable and also asymptotically free [3]. In other words the consequences of this characteristic of non-Abelian gauge theories could be explored in a simple environment without the complications of the gauge structure. One reason why higher-dimensional theories might be of interest for lowerdimensional ones is that the ultraviolet behavior of one theory could be related to the infrared dynamics of another $[4,5]$. That idea has yet to be realized concretely in the gauge theory context. However in the case of scalar $\phi^{3}$ theory, where the calculations were easier to carry out, it has been used to explore certain infrared ideas associated with the strong interactions. For instance, it has proved useful as a toy model of Regge theory but in six dimensions where ladder diagrams were analyzed in order to gain insight into the Regge slope. Several articles in this direction in $\phi^{3}$ theory are [6-8], for example. Clearly there are limitations to such exercises. The obvious one is that of an unphysical spacetime dimension. A more serious one is

Published by the American Physical Society under the terms of the Creative Commons Attribution 4.0 International license. Further distribution of this work must maintain attribution to the author(s) and the published article's title, journal citation, and DOI. Funded by SCOAP ${ }^{3}$. the lack of a bounded Hamiltonian which means that true bound state analyses could not be fully credible. Despite this the theory played a valuable role as a sounding board for exploring new ideas in a gauge theory. Other applications of cubic scalar theory lie mainly in condensed matter physics and in particular critical phenomena. For instance various decorations of the scalar field with different symmetries allowed the critical exponents that relate to percolation and the Lee-Yang edge singularity problems [9-11] to be determined very accurately in the $\epsilon$ expansion in integer dimensions below six.

More recently another perhaps surprising example of the connection a cubic scalar theory has with physics has emerged. In [12,13] the idea that on-shell gravity could be interpreted as a double copy of Yang-Mills theory was initially put forward and generated a large amount of interest. For instance it was shown that there was a relation between the product of Yang-Mills $n$-point functions and the corresponding on-shell gravity amplitudes representing a connection with the Kawai-Lewellen-Tye relations [14]. This double copy of Yang-Mills theory appears to be widely accepted as an interesting interpretation and clearly is a direction to pursue in the quest for a theory of quantum gravity. One consequence of the double copy vision was the connection with a scalar field endowed with a bi-adjoint symmetry, $[15,16]$, although the gravity connection with a scalar cubic interaction was observed earlier in [17]. For instance in [15] it was shown that scattering amplitudes of the double copy theory could be related to the gluonic ones in pure Yang-Mills theory. Another direction that was followed in $[18,19]$ was to study classical solutions of a linearized version of Yang-Mills theory and their relation to double copies of scalar fields in the bi-adjoint cubic theory. These ideas were explored further in $[20,21]$ where new 
solutions were found further strengthening the double copy correspondence concept.

While most of these studies were classical it is worth investigating the underlying quantum field theory in its own right to ascertain whether it has any other interesting properties. That is the purpose of this article. In particular we will renormalize the theory to four loops in six dimensions and deduce the renormalization group functions. Doing so will reveal a curious feature. It will transpire that for general Lie groups the one-loop coefficient of the $\beta$ function vanishes despite there being a one-loop contribution to the 3-point vertex function. This is a rather unusual and rare situation in a renormalizable theory although it is known that the one-loop term of renormalization group functions, other than the $\beta$ function, can be zero in other models. What makes this biadjoint theory even more intriguing is that the nonzero twoloop $\beta$-function coefficient is negative. Therefore this model appears to be one of the few cases where the property of asymptotic freedom is determined purely from the two-loop term of the $\beta$ function. We will explore some of the basic consequences of this property as well as finding the underlying reason why it emerges. As part of this investigation we will renormalize a generalization of the bi-adjoint model by allowing the field to take values in the adjoint representation of four (different) Lie groups which we will refer to as the quartic adjoint model.

The article is organized as follows. The background to the properties of the bi-adjoint cubic scalar theory such as the group theory connected with the computation is discussed in Sec. II. That will also include the details of how we performed the computation, the results of which are discussed in Sec. III. The generalization to the quartic adjoint model is provided in the next section before the concluding remarks of Sec. V. An appendix records full details of the renormalization group functions of the quartic adjoint model.

\section{BACKGROUND}

To begin with we define the six-dimensional Lagrangian for the bi-adjoint cubic scalar theory that was derived from solutions of linearized Yang-Mills theory and related to the double copy of gravity. If we denote the basic scalar field by $\phi^{a_{1} a_{2}}$ then the Lagrangian is, for example from $[19,20]$,

$L=\frac{1}{2}\left(\partial_{\mu} \phi^{a_{1} a_{2}}\right)^{2}+\frac{g}{6} f^{a_{1} b_{1} c_{1}} f^{a_{2} b_{2} c_{2}} \phi^{a_{1} a_{2}} \phi^{b_{1} b_{2}} \phi^{c_{1} c_{2}}$.

Given that the fields take values in the group $G_{1} \times G_{2}$, we use a different notation from $[19,20]$ as we will carry out a more general analysis subsequently. Therefore we note that the numerical label on the adjoint indices will correspond to the label on the respective subgroups in the overall symmetry group. The roman letter that carries that label is the one that is summed over in any repetition. Moreover these indices will run over a set whose dimension is the dimension of the adjoint representation of the respective group and denoted by $N_{i}$. So for instance $1 \leq a_{1} \leq N_{1}$ and
$1 \leq b_{2} \leq N_{2}$ or in more general terms $1 \leq a_{i}, b_{i}, c_{i}, d_{i} \leq$ $N_{i}$ for each $i$. In other words

$$
\delta^{a_{i} a_{i}}=N_{i}
$$

where there is never a sum over the repeated label $i$ which indicates the specific group. If $G_{1}=S U\left(N_{c}\right)$ for example then $N_{1}=N_{c}^{2}-1$ corresponding to the dimension of the adjoint representation. The respective structure functions of each group appearing in (2.1) are $f^{a_{i} b_{i} c_{i}}$ for $i=1$ and 2. As we will be carrying out loop computations it is worth discussing related group theory quantities that will appear later. For example, we use a compact notation for the Casimirs of each with

$$
f^{a_{i} c_{i} d_{i}} f^{b_{i} c_{i} d_{i}}=C_{i} \delta^{a_{i} b_{i}}
$$

for each $i$. Ordinarily one uses $C_{A}$ or $C_{2}(G)$ where $A$ denotes the adjoint representation of the group $G$ for what we now denote by $C_{i}$. It is not necessary however to include the representation designation since the adjoint will be used throughout the article unless stated otherwise. Beyond the first few loop orders higher rank group Casimirs will appear. This was noted when the four-loop $\beta$ function of QCD was determined in [22] and a comprehensive study was undertaken in [23] of general Lie group Casimirs in the context of perturbative computations. We briefly summarize the relevant aspects that are needed here. For instance the fully symmetric rank 4 tensor defined by [23]

$$
d_{R_{i}}^{a_{i} b_{i} c_{i} d_{i}}=\frac{1}{6} \operatorname{Tr}\left(T_{R_{i}}^{a_{i}} T_{R_{i}}^{\left(b_{i}\right.} T_{R_{i}}^{c_{i}} T_{R_{i}}^{\left.d_{i}\right)}\right)
$$

will arise. Here we revert momentarily to representation $R_{i}$ of the group $G_{i}$ as (2.4) involves the group generators $T_{R_{i}}^{a_{i}}$. Within the computation the contracted product of these tensors will produce additional group Casmirs independent of $C_{i}$. At four loops in Yang-Mills theory the only combination that appears turns out to be the simple product [23]

$$
d_{(i) 44}=d_{A}^{a_{i} b_{i} c_{i} d_{i}} d_{A}^{a_{i} b_{i} c_{i} d_{i}}
$$

in our notation where the bracketed label is used to avoid any potential ambiguity with the tensor rank when products of more groups are considered and $A$ denotes the adjoint representation. In the four-loop QCD $\beta$ function [23] by contrast, products of $d_{R_{i}}^{a_{i} b_{i} c_{i} d_{i}}$ in the fundamental and adjoint representations also arise. As a reference point for results that appear later we recall that for $S U\left(N_{c}\right)$ [23]

$$
d_{(i) 44}=\frac{N_{c}^{2}\left[N_{c}^{2}+36\right]}{24} N_{i}
$$

and we have not substituted the explicit value for $N_{i}$ as that quantity appears in the results for a general Lie group which is what we use throughout. Higher rank tensors beyond (2.4) have been discussed in [23]. A secondary motivation for 
studying the renormalization of (2.1) at large loop order is to ascertain whether such rank 4 tensor Casimirs first appear at the same loop order as that of QCD or not. In terms of other aspects of (2.1) for ease of comparison we retain the conventions that were used in [24]. In particular in [24] the sign of the coupling constant $g$ was opposite to that used in earlier work by others such as [3,25-27]. There ought not to be difficulty in translating where necessary.

Before renormalizing (2.1) we recall our notation. First if we denote bare entities with a subscript o then their relation to the renormalized counterparts are

$$
\phi_{\mathrm{o}}^{a_{1} a_{2}}=\sqrt{Z_{\phi}} \phi^{a_{1} a_{2}}, \quad g_{\mathrm{o}}=Z_{g} g
$$

in six dimensions. However we will dimensionally regularize (2.1) in $d=6-2 \epsilon$ dimensions and determine the renormalization group functions in the $\overline{\mathrm{MS}}$ scheme. To find $Z_{\phi}$ and $Z_{g}$ to four-loop order we have to compute the 2- and 3 -point functions and we will follow the algorithm used in [24] where more details of the technicalities of this exercise can be found. However given the presence of the structure constants in the interaction, we have had to adapt that method to determine $Z_{g}$ in particular. For example there are 540 Feynman graphs to evaluate for the four-loop vertex function which is a large number to handle. To circumvent this a shortcut was exploited which was to generate these and lower-loop vertex graphs from the 2-point graphs by applying a simple mapping to each propagator. In other words setting

$$
\frac{1}{k^{2}} \rightarrow \frac{1}{k^{2}}+\frac{\lambda}{\left(k^{2}\right)^{2}}
$$

and retaining only terms with zero or one power of the parameter $\lambda$, means that the $O(\lambda)$ terms will formally correspond to 3-point function graphs where one external momentum is set to zero [24]. In six dimensions this is infrared safe and evaluating all the graphs that are $O(\lambda)$ will produce the full 3-point function to that loop order. While this simple mapping is the essence of what one needs to do at the level of the graph generation, it is not sufficient for (2.1) as the group theory factors need to be accommodated. To achieve this we adapt (2.8) by including the group structure of the propagator as well as the inserted vertex to produce the mapping for (2.1)

$\frac{1}{k^{2}} \delta^{a_{1} b_{1}} \delta^{a_{2} b_{2}} \rightarrow \frac{1}{k^{2}} \delta^{a_{1} b_{1}} \delta^{a_{2} b_{2}}+\frac{\lambda g}{\left(k^{2}\right)^{2}} f^{a_{1} b_{1} c_{1}^{e}} f^{a_{2} b_{2} c_{2}^{e}}$

where $c_{i}^{e}$ are the external indices of the inserted leg of the generated 3-point function. One benefit of using this technique is that there are only 64 four-loop graphs in the 2-point function and the insertion does not change the underlying graph topology. This is important and leads to a more efficient computation since the same integration subroutine for that topology can be used to determine the divergences of the 2-point graph and its associated 3-point one where there is a nullified insertion on each propagator. While we have not included a mass term in (2.1) we can still compute the anomalous dimension of the mass by inserting the mass operator $\frac{1}{2} \phi^{a_{1} a_{2}} \phi^{a_{1} a_{2}}$ in a 2-point function. The renormalization constant associated with this operator is equivalent to that of the mass itself which we denote by $Z_{m}$. Therefore we can directly use (2.8) to determine $Z_{m}$ as well as $Z_{g}$.

The computational strategy to evaluate the graphs of the 2- and 3-point functions is to use the Laporta integration by parts algorithm, [28]. This constructs relations between a set of Feynman integrals that can be algebraically solved in such a way that all the integrals are related to a relatively small set. These are termed the master integrals and have to be evaluated directly. In our case since all the 2-point fourloop master integrals are available in four dimensions [29] it was possible to connect these to the ones that emerge in our six-dimensional computation [24]. This is achieved by the Tarasov method $[30,31]$ whereby integrals in $d$ dimensions can be related to the $(d+2)$-dimesional integral with the same topology and other topologies where one or more edges have been removed. Therefore the four-loop sixdimensional masters were deduced in [24].

To effect the Laporta algorithm we used the REDUZE implementation [32,33]. A useful feature of the package is that it allows the database that is generated to be written in the symbolic language FORM [34,35]. This is important since we have written an automatic program in FORM to carry out the full computation. In particular the contributing Feynman graphs are generated with the QGRAF package [36] and the topology mapping appended. This allows the automatic program to proceed since the integration of each topology follows a separate path. The final stage is the summation of all the graphs and the implementation of the automatic renormalization to deduce $Z_{\phi}, Z_{m}$ and $Z_{g}$. To do this we follow [37] which means that the graphs are evaluated with bare parameters with the renormalization constants (2.7) being introduced at the end. For instance the 2-point function is multiplied overall by $Z_{\phi}$ which allows one to deduce the unknown counterterms. Equally for the extraction of the mass and coupling constant renormalization constants the parameter $\lambda$ in each of (2.8) and (2.9) are multiplied by $Z_{m}$ and $Z_{g} \sqrt{Z_{\phi}}$ respectively. One major tool that was used to carry out the manipulation of the large number of structure functions present at each vertex was the color. h package written in FORM and available from [34]. It encodes the group theory discussed in [23] in an efficient way particularly for the three- and four-loop graphs.

\section{RESULTS}

Having outlined our computational strategy we can now record the outcome of determining $Z_{\phi}, Z_{m}$ and $Z_{g}$ that lead to the respective renormalization group functions $\gamma_{\phi}(g)$, $\gamma_{m}(g)$ and $\beta(g)$ in the $\overline{\mathrm{MS}}$ scheme. First the anomalous dimension of the scalar field is 


$$
\begin{aligned}
\gamma_{\phi}(g)= & -\frac{g^{2}}{12} C_{1} C_{2}-\frac{5 g^{4}}{432} C_{1}^{2} C_{2}^{2}-\frac{827 g^{6}}{248832} C_{1}^{3} C_{2}^{3}+\left[1032 \zeta_{3} C_{1}^{4} C_{2}^{4} N_{1} N_{2}+108 \zeta_{4} C_{1}^{4} C_{2}^{4} N_{1} N_{2}-960 \zeta_{5} C_{1}^{4} C_{2}^{4} N_{1} N_{2}\right. \\
& -943 C_{1}^{4} C_{2}^{4} N_{1} N_{2}-576 \zeta_{3} C_{1}^{4} d_{(2) 44} N_{1}-2592 \zeta_{4} C_{1}^{4} d_{(2) 44} N_{1}-11520 \zeta_{5} C_{1}^{4} d_{(2) 44} N_{1}+14976 C_{1}^{4} d_{(2) 44} N_{1} \\
& -576 \zeta_{3} C_{2}^{4} d_{(1) 44} N_{2}-2592 \zeta_{4} C_{2}^{4} d_{(1) 44} N_{2}-11520 \zeta_{5} C_{2}^{4} d_{(1) 44} N_{2}+14976 C_{2}^{4} d_{(1) 44} N_{2}+96768 \zeta_{3} d_{(1) 44} d_{(2) 44} \\
& \left.+62208 \zeta_{4} d_{(1) 44} d_{(2) 44}-138240 \zeta_{5} d_{(1) 44} d_{(2) 44}\right] \frac{g^{8}}{497664 N_{1} N_{2}}+O\left(g^{10}\right)
\end{aligned}
$$

where $\zeta_{z}$ denotes the Riemann zeta function and we note that the rank 4 group tensors first appear at four loops. For the mass anomalous dimension we find

$$
\begin{aligned}
\gamma_{m}(g)= & -\frac{g^{2}}{2} C_{1} C_{2}+\frac{5 g^{4}}{48} C_{1}^{2} C_{2}^{2}+\left[432 \zeta_{3}-2203\right] \frac{C_{1}^{3} C_{2}^{3} g^{6}}{13824}+\left[127764 \zeta_{3} C_{1}^{4} C_{2}^{4} N_{1} N_{2}+1944 \zeta_{4} C_{1}^{4} C_{2}^{4} N_{1} N_{2}\right. \\
& -251640 \zeta_{5} C_{1}^{4} C_{2}^{4} N_{1} N_{2}+255517 C_{1}^{4} C_{2}^{4} N_{1} N_{2}+88128 \zeta_{3} C_{1}^{4} d_{(2) 44} N_{1}-46656 \zeta_{4} C_{1}^{4} d_{(2) 44} N_{1} \\
& -492480 \zeta_{5} C_{1}^{4} d_{(2) 44} N_{1}+409536 C_{1}^{4} d_{(2) 44} N_{1}+88128 \zeta_{3} C_{2}^{4} d_{(1) 44} N_{2}-46656 \zeta_{4} C_{2}^{4} d_{(1) 44} N_{2} \\
& -492480 \zeta_{5} C_{2}^{4} d_{(1) 44} N_{2}+409536 C_{2}^{4} d_{(1) 44} N_{2}+3110400 \zeta_{3} d_{(1) 44} d_{(2) 44}+1119744 \zeta_{4} d_{(1) 44} d_{(2) 44} \\
& \left.+622080 \zeta_{5} d_{(1) 44} d_{(2) 44}\right] \frac{g^{8}}{1492992 N_{1} N_{2}}+O\left(g^{10}\right)
\end{aligned}
$$

where the higher order Casimirs first appear at the same order.

To complete the set, the four-loop $\beta$ function is

$$
\begin{aligned}
\beta(g)= & -\frac{5 g^{5}}{1152} C_{1}^{2} C_{2}^{2}+\left[108 \zeta_{3} C_{1}^{4} C_{2}^{4} N_{1} N_{2}-161 C_{1}^{4} C_{2}^{4} N_{1} N_{2}-2592 \zeta_{3} C_{1}^{4} d_{(2) 44} N_{1}+2592 C_{1}^{4} d_{(2) 44} N_{1}\right. \\
& \left.-2592 \zeta_{3} C_{2}^{4} d_{(1) 44} N_{2}+2592 C_{2}^{4} d_{(1) 44} N_{2}+62208 \zeta_{3} d_{(1) 44} d_{(2) 44}\right] \frac{g^{7}}{41472 C_{1} C_{2} N_{1} N_{2}} \\
& +\left[-368928 \zeta_{3} C_{1}^{4} C_{2}^{4} N_{1} N_{2}+518400 \zeta_{5} C_{1}^{4} C_{2}^{4} N_{1} N_{2}-101089 C_{1}^{4} C_{2}^{4} N_{1} N_{2}\right. \\
& -3483648 \zeta_{3} C_{1}^{4} d_{(2) 44} N_{1}+6220800 \zeta_{5} C_{1}^{4} d_{(2) 44} N_{1}-2363904 C_{1}^{4} d_{(2) 44} N_{1} \\
& -3483648 \zeta_{3} C_{2}^{4} d_{(1) 44} N_{2}+6220800 \zeta_{5} C_{2}^{4} d_{(1) 44} N_{2}-2363904 C_{2}^{4} d_{(1) 44} N_{2} \\
& -95551488 \zeta_{3} d_{(1) 44} d_{(2) 44}+119439360 \zeta_{5} d_{(1) 44} d_{(2) 44} \frac{g^{9}}{23887872 N_{1} N_{2}}+O\left(g^{11}\right) .
\end{aligned}
$$

This has the unusual feature in that the first nonzero term is at two loops rather than one loop. This is not the first or only case of the first term of a renormalization group function in a fully renormalizable field theory being absent. For instance, while the field anomalous dimension in four-dimensional $\phi^{4}$ theory is zero at one loop this is for the simple reason that the only graph contributing to the 2-point function is a snail. Therefore it is independent of the external momentum and its divergence contributes to the mass renormalization only. Here the situation is different in that the only one-loop graph of the 3-point vertex is divergent but the residue of the simple pole is exactly cancelled by the contribution from the wave function renormalization. This is not the case for other symmetry decorations of the scalar field in scalar $\phi^{3}$ theory in six dimensions $[3,26,27]$. This curious property has an interesting consequence which is that since the coefficient of the now leading two-loop term of $\beta(g)$ is negative then the theory is asymptotically free. Ordinarily when this is a feature of other field theories it is purely from the one-loop term $[1,2]$. We note at this point that this sign of the two-loop term would have emerged irrespective of the coupling constant sign convention alluded to earlier. One comment that deserves mention at this point concerns the scheme dependence of this particular $\beta$ function. Even though the one-loop term is zero the three-loop term of $\beta(g)$ still depends on the renormalization scheme. Unlike the other two renormalization group functions the rank 4 Casimirs first appear at three loops in the $\beta$ function rather than four. This is one order earlier than that of QCD [22].

To gain more insight into the consequences of there being no one-loop term of the $\beta$ function, it is worth focusing on the case when both groups $G_{1}$ and $G_{2}$ are the same which we will denote by $G$. In this case (3.1), (3.2) and (3.3) become 


$$
\begin{aligned}
\gamma_{\phi}^{G \times G}(g)= & -\frac{C_{1}^{2} g^{2}}{12}-\frac{5 C_{1}^{4} g^{4}}{432}-\frac{827 C_{1}^{6} g^{6}}{248832}+\left[1032 \zeta_{3} C_{1}^{8} N_{1}^{2}-1152 \zeta_{3} C_{1}^{4} d_{(1) 44} N_{1}+96768 \zeta_{3} d_{(1) 44}^{2}+108 \zeta_{4} C_{1}^{8} N_{1}^{2}\right. \\
& -5184 \zeta_{4} C_{1}^{4} d_{(1) 44} N_{1}+62208 \zeta_{4} d_{(1) 44}^{2}-960 \zeta_{5} C_{1}^{8} N_{1}^{2}-23040 \zeta_{5} C_{1}^{4} d_{(1) 44} N_{1}-138240 \zeta_{5} d_{(1) 44}^{2}-943 C_{1}^{8} N_{1}^{2} \\
& \left.+29952 C_{1}^{4} d_{(1) 44} N_{1}\right] \frac{g^{8}}{497664 N_{1}^{2}}+O\left(g^{10}\right), \\
\gamma_{m}^{G \times G}(g)= & -\frac{C_{1}^{2} g^{2}}{2}+\frac{5 C_{1}^{4} g^{4}}{48}+C_{1}^{6}\left[432 \zeta_{3}-2203\right] \frac{g^{6}}{13824}+\left[127764 \zeta_{3} C_{1}^{8} N_{1}^{2}+176256 \zeta_{3} C_{1}^{4} d_{(1) 44} N_{1}+3110400 \zeta_{3} d_{(1) 44}^{2}\right. \\
& +1944 \zeta_{4} C_{1}^{8} N_{1}^{2}-93312 \zeta_{4} C_{1}^{4} d_{(1) 44} N_{1}+1119744 \zeta_{4} d_{(1) 44}^{2}-251640 \zeta_{5} C_{1}^{8} N_{1}^{2}-984960 \zeta_{5} C_{1}^{4} d_{(1) 44} N_{1} \\
& \left.+622080 \zeta_{5} d_{(1) 44}^{2}+255517 C_{1}^{8} N_{1}^{2}+819072 C_{1}^{4} d_{(1) 44} N_{1}\right] \frac{g^{8}}{1492992 N_{1}^{2}}+O\left(g^{10}\right)
\end{aligned}
$$

and

$$
\begin{aligned}
\beta^{G \times G}(g)= & -\frac{5 C_{1}^{4} g^{5}}{1152}+\left[108 \zeta_{3} C_{1}^{8} N_{1}^{2}-5184 \zeta_{3} C_{1}^{4} d_{(1) 44} N_{1}+62208 \zeta_{3} d_{(1) 44}^{2}-161 C_{1}^{8} N_{1}^{2}+5184 C_{1}^{4} d_{(1) 44} N_{1}\right] \frac{g^{7}}{41472 C_{1}^{2} N_{1}^{2}} \\
& +\left[-368928 \zeta_{3} C_{1}^{8} N_{1}^{2}-6967296 \zeta_{3} C_{1}^{4} d_{(1) 44} N_{1}-95551488 \zeta_{3} d_{(1) 44}^{2}+518400 \zeta_{5} C_{1}^{8} N_{1}^{2}+12441600 \zeta_{5} C_{1}^{4} d_{(1) 44} N_{1}\right. \\
& \left.+119439360 \zeta_{5} d_{(1) 44}^{2}-101089 C_{1}^{8} N_{1}^{2}-4727808 C_{1}^{4} d_{(1) 44} N_{1}\right] \frac{g^{9}}{23887872 N_{1}^{2}}+O\left(g^{11}\right)
\end{aligned}
$$

Specifying to the group $S U(3)$ we deduce

$$
\begin{aligned}
& \gamma_{\phi}^{S U(3) \times S U(3)}(g)=-\frac{3}{42} g^{2}-\frac{15}{16} g^{4}-\frac{2481}{1024} g^{6}+27\left[4992 \zeta_{3}+1728 \zeta_{4}-11760 \zeta_{5}+5297\right] \frac{g^{8}}{2048}+O\left(g^{10}\right) \\
& \gamma_{m}^{S U(3) \times S U(3)}(g)=-\frac{9}{2} g^{2}+\frac{135}{16} g^{4}+27\left[432 \zeta_{3}-2203\right] \frac{g^{6}}{512}+9\left[299484 \zeta_{3}+31104 \zeta_{4}-429840 \zeta_{5}+426157\right] \frac{g^{8}}{2048}+O\left(g^{10}\right), \\
& \beta^{S U(3) \times S U(3)}(g)=-\frac{45}{128} g^{5}+9\left[1728 \zeta_{3}+919\right] \frac{g^{7}}{512}+9\left[8294400 \zeta_{5}-5967648 \zeta_{3}-1086049\right] \frac{g^{9}}{32768}+O\left(g^{11}\right)
\end{aligned}
$$

or

$$
\begin{aligned}
& \gamma_{\phi}^{S U(3) \times S U(3)}(g)=-0.750000 g^{2}-0.937500 g^{4}-2.422852 g^{6}+12.836235 g^{8}+O\left(g^{10}\right), \\
& \gamma_{m}^{S U(3) \times S U(3)}(g)=-4.500000 g^{2}+8.437500 g^{4}-88.789469 g^{6}+1644.017718 g^{8}+O\left(g^{10}\right), \\
& \beta^{S U(3) \times S U(3)}(g)=-0.351562 g^{5}+52.666775 g^{7}+93.711209 g^{9}+O\left(g^{11}\right)
\end{aligned}
$$

numerically. From (3.8) it is clear that there is a Banks-Zaks fixed point [38] stemming from the opposite signs of the first two terms of the $\beta$ function. In fact it is also the case that a similar fixed point is present for $S U(M) \times S U(N)$. Strictly the fixed point of [38] in QCD derives from the one- and two-loop scheme independent terms. We use it here in the sense of the first two nonzero terms although the second of these is scheme dependent unlike [38]. Moreover the value of the critical coupling for $N=3$ only changes by around $1 \%$ when solving $\beta^{S U(3) \times S U(3)}(g)=0$ at three and four loops. One of the reasons for providing this example is to show another interesting consequence of the absence of the one-loop term. While the renormalization group functions are scheme dependent, one can derive renormalization group invariants from them. These are critical exponents that are the evaluation of the functions at a nontrivial fixed point. One important such fixed point is the Wilson-Fisher one $[39,40]$, where the critical coupling is defined by setting the $d$-dimensional $\beta$ function to zero and denoted by $g_{*}$. So in $d=6-2 \epsilon$ dimensions we have 


$$
\begin{aligned}
\eta^{S U(3) \times S U(3)=} & -\frac{2 i \sqrt{5}}{5} \sqrt{\epsilon}+\frac{2}{25}\left[576 \zeta_{3}+323\right] \epsilon+\sqrt{5} i\left[1327104 \zeta_{3}^{2}+779232 \zeta_{3}+921600 \zeta_{5}+271945\right] \frac{\epsilon^{\frac{3}{2}}}{500} \\
& +\left[311040 \zeta_{4}-143327232 \zeta_{3}^{2}-96163200 \zeta_{3}-85060800 \zeta_{5}-31005017\right] \frac{\epsilon^{2}}{6750}+O\left(\epsilon^{\frac{5}{2}}\right) \\
\eta_{m}^{S U(3) \times S U(3)}= & -\frac{12 i \sqrt{5}}{5} \sqrt{\epsilon}+16\left[432 \zeta_{3}+211\right] \frac{\epsilon}{25}+\frac{9 i \sqrt{5}}{250}\left[442368 \zeta_{3}^{2}+233664 \zeta_{3}+307200 \zeta_{5}+79175\right] \epsilon^{\frac{3}{2}} \\
& +2\left[110854656 \zeta_{3}^{2}+55734372 \zeta_{3}+155520 \zeta_{4}+60058800 \zeta_{5}+15279107\right] \frac{\epsilon^{2}}{1125}+O\left(\epsilon^{\frac{5}{2}}\right), \\
\omega^{S U(3) \times S U(3)}= & 2 \epsilon-\frac{2 i \sqrt{5}}{5}\left[1728 \zeta_{3}+919\right] \epsilon^{\frac{3}{2}}+\left[35831808 \zeta_{3}^{2}+8274528 \zeta_{3}+41472000 \zeta_{5}+4704487\right] \frac{\epsilon^{2}}{2250}+O\left(\epsilon^{\frac{5}{2}}\right)
\end{aligned}
$$

where $\eta=\gamma_{\phi}\left(g_{*}\right), \eta_{m}=\gamma_{m}\left(g_{*}\right)$ and $\omega=2 \beta^{\prime}\left(g_{*}\right)$. The main key difference between these exponents and those from models where there is a nonzero one-loop term is that the expansion is a function of $\sqrt{\epsilon}$ rather than $\epsilon$. In addition the exponents are complex but this is due to having assumed $\epsilon$ is real and positive. If $\epsilon$ were real and negative then the exponents are real above six dimensions.

Finally we close this section by recalling that solving the $\beta$ function as a differential equation determines the functional dependence of the running coupling constant with the renormalization scale $\mu$. Therefore we can compare the running coupling constants in the conventional case where asymptotic freedom is determined by the one-loop $\beta$ function with that for (2.1). For instance, if we formally define two $\beta$ functions by

$$
\begin{aligned}
& \beta_{1}\left(g_{1}\right)=-\bar{\beta}_{1} g_{1}^{3}+O\left(g_{1}^{5}\right), \\
& \beta_{2}\left(g_{2}\right)=-\bar{\beta}_{2} g_{2}^{5}+O\left(g_{2}^{7}\right)
\end{aligned}
$$

then we have

$$
g_{1}^{2}(\mu)=-\frac{1}{\bar{\beta}_{1} \ln \left(\mu^{2} / \Lambda_{1}^{2}\right)}
$$

for the more conventional one-loop $\beta$ function. By contrast solving the second case we find

$$
g_{2}^{2}(\mu)=-\frac{1}{\sqrt{2 \bar{\beta}_{2} \ln \left(\mu^{2} / \Lambda_{2}^{2}\right)}}
$$

where $\Lambda_{1}$ and $\Lambda_{2}$ are the constants of integration. Clearly both running coupling constants have the same general behavior in that they tend to zero as $\mu \rightarrow \infty$. However in the latter case where the one-loop $\beta$-function term is absent, the coupling constant tends to zero at a much slower rate. So if this model, or one with the same property, was realized in Nature the constituent particles would only be effectively free at significantly high energies.

\section{QUARTIC ADJOINT}

The absence of a one-loop term in the $\beta$ function of (2.1) is an interesting property. In order to see whether this property is common to more general scalar $\phi^{3}$ theories with adjoint decorations, we have repeated the renormalization exercise for (2.1) for what we will term the quartic adjoint theory with Lagrangian

$$
L=\frac{1}{2}\left(\partial_{\mu} \phi^{a_{1} a_{2} a_{3} a_{4}}\right)^{2}+\frac{g}{6} f^{a_{1} b_{1} c_{1}} f^{a_{2} b_{2} c_{2}} f^{a_{3} b_{3} c_{3}} f^{a_{4} b_{4} c_{4}} \boldsymbol{\phi}^{a_{1} a_{2} a_{3} a_{4}} \boldsymbol{\phi}^{b_{1} b_{2} b_{3} b_{4}} \boldsymbol{\phi}^{c_{1} c_{2} c_{3} c_{4}} .
$$

Here we have a scalar field which takes values in the group $G_{1} \times G_{2} \times G_{3} \times G_{4}$ and in particular the interaction involves the adjoint representation of the group generators. In (4.1) we use a similar notation to that introduced for (2.1) where there are now two additional labels due to the extra groups $G_{3}$ and $G_{4}$. Equally the definition of the group Casimirs have an obvious natural extension of those given in (2.2), (2.3) and (2.5). We have followed the same process of renormalizing (4.1) as that for (2.1) together with similar consistency checks. Therefore we move to the discussion of the outcome. With the additional group structure it transpires that the four-loop expressions for each of the renormalization group functions are more involved than those of (2.1). These have been recorded in the Appendix. Instead we illustrate the structure in the simpler case of the group $G \times G \times G \times G \equiv G^{4}$ and find 


$$
\begin{aligned}
\gamma_{\phi}^{G^{4}}(g)= & -\frac{C_{1}^{4} g^{2}}{12}-\frac{19 C_{1}^{8} g^{4}}{864}-\frac{40421 C_{1}^{12} g^{6}}{3981312}+\left[1910 \zeta_{3} C_{1}^{16} N_{1}^{4}+2112 \zeta_{3} C_{1}^{12} d_{(1) 44} N_{1}^{3}+89856 \zeta_{3} C_{1}^{8} d_{(1) 44}^{2} N_{1}^{2}\right. \\
& -110592 \zeta_{3} C_{1}^{4} d_{(1) 44}^{3} N_{1}+4644864 \zeta_{3} d_{(1) 44}^{4}+9 \zeta_{4} C_{1}^{16} N_{1}^{4}-864 \zeta_{4} C_{1}^{12} d_{(1) 44} N_{1}^{3}+31104 \zeta_{4} C_{1}^{8} d_{(1) 44}^{2} N_{1}^{2} \\
& -497664 \zeta_{4} C_{1}^{4} d_{(1) 44}^{3} N_{1}+2985984 \zeta_{4} d_{(1) 44}^{4}-320 \zeta_{5} C_{1}^{16} N_{1}^{4}-15360 \zeta_{5} C_{1}^{12} d_{(1) 44} N_{1}^{3}-276480 \zeta_{5} C_{1}^{8} d_{(1) 44}^{2} N_{1}^{2} \\
& -2211840 \zeta_{5} C_{1}^{4} d_{(1) 44}^{3} N_{1}-6635520 \zeta_{5} d_{(1) 44}^{4}-150934 C_{1}^{16} N_{1}^{4}+14976 C_{1}^{12} d_{(1) 44} N_{1}^{3}+179712 C_{1}^{8} d_{(1) 44}^{2} N_{1}^{2} \\
& \left.+2875392 C_{1}^{4} d_{(1) 44}^{3} N_{1}\right] \frac{g^{8}}{23887872 N_{1}^{4}}+O\left(g^{10}\right)
\end{aligned}
$$

and

$$
\begin{aligned}
\gamma_{m}^{G^{4}}(g)= & -\frac{C_{1}^{4} g^{2}}{2}+\frac{C_{1}^{8} g^{2}}{96}+\left[3024 \zeta_{3}-43537\right] \frac{C_{1}^{12} g^{6}}{221184}+\left[-619077 \zeta_{3} C_{1}^{16} N_{1}^{4}+58176 \zeta_{3} C_{1}^{12} d_{(1) 44} N_{1}^{3}+1389312 \zeta_{3} C_{1}^{8} d_{(1) 44}^{2} N_{1}^{2}\right. \\
& +5640192 \zeta_{3} C_{1}^{4} d_{(1) 44}^{3} N_{1}+49766400 \zeta_{3} d_{(1) 44}^{4}-91800 \zeta_{4} C_{1}^{16} N_{1}^{4}-5184 \zeta_{4} C_{1}^{12} d_{(1) 44} N_{1}^{3}+186624 \zeta_{4} C_{1}^{8} d_{(1) 44}^{2} N_{1}^{2} \\
& -2985984 \zeta_{4} C_{1}^{4} d_{(1) 44}^{3} N_{1}+17915904 \zeta_{4} d_{(1) 44}^{4}-177630 \zeta_{5} C_{1}^{16} N_{1}^{4}-158400 \zeta_{5} C_{1}^{12} d_{(1) 44} N_{1}^{3} \\
& -1762560 \zeta_{5} C_{1}^{8} d_{(1) 44}^{2} N_{1}^{2}-31518720 \zeta_{5} C_{1}^{4} d_{(1) 44}^{3} N_{1}+9953280 \zeta_{5} d_{(1) 44}^{4}+2070250 C_{1}^{16} N_{1}^{4}+136512 C_{1}^{12} d_{(1) 44} N_{1}^{3} \\
& \left.+1638144 C_{1}^{8} d_{(1) 44}^{2} N_{1}^{2}+26210304 C_{1}^{4} d_{(1) 44}^{3} N_{1}\right] \frac{g^{8}}{23887872 N_{1}^{4}}+O\left(g^{10}\right)
\end{aligned}
$$

for the field and mass anomalous dimensions. For the $\beta$ function we arrived at

$$
\begin{aligned}
\beta^{G^{4}}(g)= & -\frac{3 C_{1}^{4} g^{3}}{32}-\frac{467 C_{1}^{8} g^{5}}{18432}+\left[48 \zeta_{3} C_{1}^{16} N_{1}^{4}-4608 \zeta_{3} C_{1}^{12} d_{(1) 44} N_{1}^{3}+165888 \zeta_{3} C_{1}^{8} d_{(1) 44}^{2} N_{1}^{2}-2654208 \zeta_{3} C_{1}^{4} d_{(1) 44}^{3} N_{1}\right. \\
& +15925248 \zeta_{3} d_{(1) 44}^{4}-125981 C_{1}^{16} N_{1}^{4}+13824 C_{1}^{12} d_{(1) 44} N_{1}^{3}+165888 C_{1}^{8} d_{(1) 44}^{2} N_{1}^{2} \\
& \left.+2654208 C_{1}^{4} d_{(1) 44}^{3} N_{1}\right] \frac{g^{7}}{10616832 C_{1}^{4} N_{1}^{4}}+\left[394304 \zeta_{3} C_{1}^{16} N_{1}^{4}-21494784 \zeta_{3} C_{1}^{12} d_{(1) 44} N_{1}^{3}-224169984 \zeta_{3} C_{1}^{8} d_{(1) 44}^{2} N_{1}^{2}\right. \\
& -7582187520 \zeta_{3} C_{1}^{4} d_{(1) 44}^{3} N_{1}-3301834752 \zeta_{3} d_{(1) 44}^{4}-15552 \zeta_{4} C_{1}^{16} N_{1}^{4}+1492992 \zeta_{4} C_{1}^{12} d_{(1) 44} N_{1}^{3} \\
& -53747712 \zeta_{4} C_{1}^{8} d_{(1) 44}^{2} N_{1}^{2}+859963392 \zeta_{4} C_{1}^{4} d_{(1) 44}^{3} N_{1}-5159780352 \zeta_{4} d_{(1) 44}^{4}+325120 \zeta_{5} C_{1}^{16} N_{1}^{4} \\
& +18370560 \zeta_{5} C_{1}^{12} d_{(1) 44} N_{1}^{3}+479969280 \zeta_{5} C_{1}^{8} d_{(1) 44}^{2} N_{1}^{2}+4636016640 \zeta_{5} C_{1}^{4} d_{(1) 44}^{3} N_{1}+24418713600 \zeta_{5} d_{(1) 44}^{4} \\
& \left.-134800515 C_{1}^{16} N_{1}^{4}+15123456 C_{1}^{12} d_{(1) 44} N_{1}^{3}+189444096 C_{1}^{8} d_{(1) 44}^{2} N_{1}^{2}+2521497600 C_{1}^{4} d_{(1) 44}^{3} N_{1}\right] \frac{g^{9}}{18345885696 N_{1}^{4}} \\
& +O\left(g^{11}\right)
\end{aligned}
$$

and there is no Banks-Zaks fixed point. By contrast to (3.3) and the parallel simplification of (3.6) we note that there is a nonzero one-loop $\beta$-function coefficient unlike the bi-adjoint model. In the general group case this coefficient is a simple product of $C_{i}$ for $i=1$ to 4 .

In order to compare with the bi-adjoint case we note that specifying to the group $S U(3)$ gives

$$
\begin{aligned}
& \gamma_{\phi}^{S U(3)^{4}}(g)=-6.750000 g^{2}-144.281250 g^{4}-5395.552185 g^{6}+2.4888387 \times 10^{5} g^{8}+O\left(g^{10}\right), \\
& \gamma_{m}^{S U(3)^{4}}(g)=-40.50000 g^{2}+68.343750 g^{4}-95872.884627 g^{6}+2.842688 \times 10^{6} g^{8}+O\left(g^{10}\right), \\
& \beta^{S U(3)^{4}}(g)=-7.593750 g^{3}-166.231934 g^{5}-458.390411 g^{7}-95378.353885 g^{9}+O\left(g^{11}\right) .
\end{aligned}
$$

With these we can illustrate the difference in the corresponding critical exponents at the Wilson-Fisher fixed point in $d=6-2 \epsilon$ dimensions by noting that 


$$
\begin{aligned}
\eta^{S U(3)^{4}=}= & \frac{4}{9} \epsilon+\frac{11}{729} \epsilon^{2}+2\left[4608 \zeta_{3}+12295\right] \frac{\epsilon^{3}}{59049}+\left[88631400 \zeta_{3}+4478976 \zeta_{4}-115795200 \zeta_{5}+33923953\right] \frac{\epsilon^{4}}{19131876} \\
& +O\left(\epsilon^{5}\right), \\
\eta_{m}^{S U(3)^{4}=}= & \frac{8}{3} \epsilon+\frac{1006}{243} \epsilon^{2}+\left[-22392 \zeta_{3}+684799\right] \frac{\epsilon^{3}}{19683}+\left[33249552 \zeta_{3}-10882512 \zeta_{4}-288886560 \zeta_{5}\right. \\
& +1436683139] \frac{\epsilon^{4}}{6377292}+O\left(\epsilon^{5}\right), \\
\omega^{S U(3)^{4}=} & \epsilon-\frac{467}{324} \epsilon^{2}+\left[-18432 \zeta_{3}+28807\right] \frac{\epsilon^{3}}{26244}+\left[-467532144 \zeta_{3}-17915904 \zeta_{4}+508069440 \zeta_{5}-192428981\right] \\
& \times \frac{\epsilon^{4}}{17006112}+O\left(\epsilon^{5}\right) .
\end{aligned}
$$

The nonzero one-loop $\beta$-function coefficient produces the standard $\epsilon$ expansion in contrast to the bi-adjoint case where the exponents depend on $\sqrt{\epsilon}$.

Having considered a second scalar theory with a group theory structure similar to that of (2.1) which does not have a zero one-loop $\beta$-function coefficient, it is worth trying to understand how this arises for (2.1). There are two parts to determining the renormalization constant $Z_{g}$ that leads to the $\beta$ function. These are the divergences from the 2- and 3-point functions. The former produces the value for $Z_{\phi}$ directly whereas the divergences of the latter do not immediately give $Z_{g}$. Instead they give the combination $Z_{g} Z_{\phi}^{\frac{3}{2}}$. So for $Z_{g}$ to have no simple pole at one loop means that the divergence from the 3-point function must exactly match that of $Z_{\phi}$ multiplied by $\frac{3}{2}$. From the explicit computation we find that the residue of the one-loop simple pole of the 2-point function is $-\frac{1}{12} C_{1} C_{2}$ whereas that for the 3-point function is $\frac{1}{8} C_{1} C_{2}$. These are clearly in the required ratio. By contrast the respective numbers for (4.1) are $-\frac{1}{12} C_{1} C_{2} C_{3} C_{4}$ and $\frac{1}{32} C_{1} C_{2} C_{3} C_{4}$. Combining these to deduce $Z_{g}$ at one loop gives the correct coefficient of $-\frac{3}{32} C_{1} C_{2} C_{3} C_{4}$ of the general $\beta$ function. Aside from the additional group theory factors the only discrepancy between both models is in the coefficient of the divergence from the 3-point function which is different by a factor of $\frac{1}{4}$. This is the origin of why (3.3) has no one-loop term and rests in the group theory deriving from the one-loop triangle graph which is the sole contribution at this loop order. Each subgroup $G_{i}$ of the symmetry group produces the combination

$$
f^{a_{i} p_{i} q_{i}} f^{b_{i} r_{i} q_{i}} f^{c_{i} p_{i} r_{i}}=\frac{1}{2} C_{i} f^{a_{i} b_{i} c_{i}}
$$

So for each subgroup this relation, derived from the Jacobi identity in the adjoint representation, gives a factor of $\frac{1}{2}$ to the residue of the simple pole of the 3-point function. As there is one factor of $\frac{1}{2}$ from the actual integration over the loop momentum then for the most general group $G_{1} \times \ldots \times G_{n}$ the 3-point function simple pole has a residue of $\frac{1}{2^{n+1}} \prod_{i=1}^{n} C_{i}$. Hence for this general group the one-loop coefficient of the $\beta$ function, denoted by $\hat{\beta}_{1}(n)$, will be

$$
\hat{\beta}_{1}(n)=\left[\frac{1}{2^{n+1}}-\frac{1}{8}\right] \prod_{i=1}^{n} C_{i}
$$

which is a monotonically decreasing function and defined for all integers $n \neq 1$. The exception is because one has a free field theory for $n=1$ since the interaction is $f^{a_{1} b_{1} c_{1}} \phi^{a_{1}} \phi^{b_{1}} \phi^{c_{1}}$ which vanishes due to the antisymmetry of the structure constants. Clearly $\hat{\beta}_{1}(2)=0$ and so the curiosity of (2.1) being asymptotically free as a consequence of the two-loop term of the $\beta$ function is purely due to a group theory property. We note that the value of $\hat{\beta}_{1}(0)$ is consistent with the known low order $\beta$ function of the pure $\phi^{3}$ theory $[3,25]$. Equally $\hat{\beta}_{1}(4)$ is in agreement with (4.4) and (A3).

\section{DISCUSSION}

Scalar $\phi^{3}$ theory has played an important role as a toy model in quantum field theory for many decades. For instance any Feynman graph generated from the basic cubic interaction can in turn generate the basic topologies that can occur in higher $n$-point interactions. This is achieved by formally deleting propagators in the graph theory sense and hence represents the initial point for combinatoric studies in quantum field theory. Where the theory has limitations in physics applications is that its critical dimension is six rather than four. However as noted earlier certain properties of scalar $\phi^{3}$ theory are similar to the more involved field theories in four dimensions and hence the six-dimensional model can be used to explore ideas. In this article we have studied an interesting modification whereby the scalar field is in a bi-adjoint representation of Lie groups. This is motivated by the double copy relation between Yang-Mills theory and on-shell gravity. While the studies of [18-21] examined classical solutions to the scalar theory, it has turned out that the six-dimensional field theory has a 
peculiar property. It is unusual that asymptotic freedom is a consequence of the two-loop term of the $\beta$ function rather than the first. However that is the case for (2.1). In studying the consequences it appears to be unique in the class of extensions that would be termed multi-adjoint as the analysis we carried out for the quartic adjoint demonstrates. It is not clear whether there is a parallel theory in four dimensions that is asymptotically free due to the two-loop $\beta$ function for which the bi-adjoint six-dimensional scalar field theory is the underlying laboratory. It was noted in [41] that a necessary condition for this is non-Abelian gauge fields.

\section{ACKNOWLEDGMENTS}

We thank Dr. T. Ryttov for discussions. This work was supported in part by a DFG Mercator Fellowship.

\section{APPENDIX: FULL RESULTS FOR QUARTIC ADJOINT}

In this appendix we record the full expressions for the renormalization group functions of the quartic adjoint scalar theory which uses similar notation to that used for the parallel expressions of the bi-adjoint case. First the field anomalous and mass anomalous dimensions are

$$
\begin{aligned}
& \gamma_{\phi}(g)=-C_{1} C_{2} C_{3} C_{4} \frac{g^{2}}{12}-19 C_{1}^{2} C_{2}^{2} C_{3}^{2} C_{4}^{2} \frac{g^{4}}{864}-40421 C_{1}^{3} C_{2}^{3} C_{3}^{3} C_{4}^{3} \frac{g^{6}}{3981312} \\
& +\left[1910 \zeta_{3} C_{1}^{4} C_{2}^{4} C_{3}^{4} C_{4}^{4} N_{1} N_{2} N_{3} N_{4}+9 \zeta_{4} C_{1}^{4} C_{2}^{4} C_{3}^{4} C_{4}^{4} N_{1} N_{2} N_{3} N_{4}\right. \\
& -320 \zeta_{5} C_{1}^{4} C_{2}^{4} C_{3}^{4} C_{4}^{4} N_{1} N_{2} N_{3} N_{4}-150934 C_{1}^{4} C_{2}^{4} C_{3}^{4} C_{4}^{4} N_{1} N_{2} N_{3} N_{4} \\
& +528 \zeta_{3} C_{1}^{4} C_{2}^{4} C_{3}^{4} d_{(4) 44} N_{1} N_{2} N_{3}-216 \zeta_{4} C_{1}^{4} C_{2}^{4} C_{3}^{4} d_{(4) 44} N_{1} N_{2} N_{3} \\
& -3840 \zeta_{5} C_{1}^{4} C_{2}^{4} C_{3}^{4} d_{(4) 44} N_{1} N_{2} N_{3}+3744 C_{1}^{4} C_{2}^{4} C_{3}^{4} d_{(4) 44} N_{1} N_{2} N_{3} \\
& +528 \zeta_{3} C_{1}^{4} C_{2}^{4} C_{4}^{4} d_{(3) 44} N_{1} N_{2} N_{4}-216 \zeta_{4} C_{1}^{4} C_{2}^{4} C_{4}^{4} d_{(3) 44} N_{1} N_{2} N_{4} \\
& -3840 \zeta_{5} C_{1}^{4} C_{2}^{4} C_{4}^{4} d_{(3) 44} N_{1} N_{2} N_{4}+3744 C_{1}^{4} C_{2}^{4} C_{4}^{4} d_{(3) 44} N_{1} N_{2} N_{4} \\
& +14976 \zeta_{3} C_{1}^{4} C_{2}^{4} d_{(3) 44} d_{(4) 44} N_{1} N_{2}+5184 \zeta_{4} C_{1}^{4} C_{2}^{4} d_{(3) 44} d_{(4) 44} N_{1} N_{2} \\
& -46080 \zeta_{5} C_{1}^{4} C_{2}^{4} d_{(3) 44} d_{(4) 44} N_{1} N_{2}+29952 C_{1}^{4} C_{2}^{4} d_{(3) 44} d_{(4) 44} N_{1} N_{2} \\
& +528 \zeta_{3} C_{1}^{4} C_{3}^{4} C_{4}^{4} d_{(2) 44} N_{1} N_{3} N_{4}-216 \zeta_{4} C_{1}^{4} C_{3}^{4} C_{4}^{4} d_{(2) 44} N_{1} N_{3} N_{4} \\
& -3840 \zeta_{5} C_{1}^{4} C_{3}^{4} C_{4}^{4} d_{(2) 44} N_{1} N_{3} N_{4}+3744 C_{1}^{4} C_{3}^{4} C_{4}^{4} d_{(2) 44} N_{1} N_{3} N_{4} \\
& +14976 \zeta_{3} C_{1}^{4} C_{3}^{4} d_{(2) 44} d_{(4) 44} N_{1} N_{3}+5184 \zeta_{4} C_{1}^{4} C_{3}^{4} d_{(2) 44} d_{(4) 44} N_{1} N_{3} \\
& -46080 \zeta_{5} C_{1}^{4} C_{3}^{4} d_{(2) 44} d_{(4) 44} N_{1} N_{3}+29952 C_{1}^{4} C_{3}^{4} d_{(2) 44} d_{(4) 44} N_{1} N_{3} \\
& +14976 \zeta_{3} C_{1}^{4} C_{4}^{4} d_{(2) 44} d_{(3) 44} N_{1} N_{4}+5184 \zeta_{4} C_{1}^{4} C_{4}^{4} d_{(2) 44} d_{(3) 44} N_{1} N_{4} \\
& -46080 \zeta_{5} C_{1}^{4} C_{4}^{4} d_{(2) 44} d_{(3) 44} N_{1} N_{4}+29952 C_{1}^{4} C_{4}^{4} d_{(2) 44} d_{(3) 44} N_{1} N_{4} \\
& -27648 \zeta_{3} C_{1}^{4} d_{(2) 44} d_{(3) 44} d_{(4) 44} N_{1}-124416 \zeta_{4} C_{1}^{4} d_{(2) 44} d_{(3) 44} d_{(4) 44} N_{1} \\
& -552960 \zeta_{5} C_{1}^{4} d_{(2) 44} d_{(3) 44} d_{(4) 44} N_{1}+718848 C_{1}^{4} d_{(2) 44} d_{(3) 44} d_{(4) 44} N_{1} \\
& +528 \zeta_{3} C_{2}^{4} C_{3}^{4} C_{4}^{4} d_{(1) 44} N_{2} N_{3} N_{4}-216 \zeta_{4} C_{2}^{4} C_{3}^{4} C_{4}^{4} d_{(1) 44} N_{2} N_{3} N_{4} \\
& -3840 \zeta_{5} C_{2}^{4} C_{3}^{4} C_{4}^{4} d_{(1) 44} N_{2} N_{3} N_{4}+3744 C_{2}^{4} C_{3}^{4} C_{4}^{4} d_{(1) 44} N_{2} N_{3} N_{4} \\
& +14976 \zeta_{3} C_{2}^{4} C_{3}^{4} d_{(1) 44} d_{(4) 44} N_{2} N_{3}+5184 \zeta_{4} C_{2}^{4} C_{3}^{4} d_{(1) 44} d_{(4) 44} N_{2} N_{3} \\
& -46080 \zeta_{5} C_{2}^{4} C_{3}^{4} d_{(1) 44} d_{(4) 44} N_{2} N_{3}+29952 C_{2}^{4} C_{3}^{4} d_{(1) 44} d_{(4) 44} N_{2} N_{3} \\
& +14976 \zeta_{3} C_{2}^{4} C_{4}^{4} d_{(1) 44} d_{(3) 44} N_{2} N_{4}+5184 \zeta_{4} C_{2}^{4} C_{4}^{4} d_{(1) 44} d_{(3) 44} N_{2} N_{4} \\
& -46080 \zeta_{5} C_{2}^{4} C_{4}^{4} d_{(1) 44} d_{(3) 44} N_{2} N_{4}+29952 C_{2}^{4} C_{4}^{4} d_{(1) 44} d_{(3) 44} N_{2} N_{4} \\
& -27648 \zeta_{3} C_{2}^{4} d_{(1) 44} d_{(3) 44} d_{(4) 44} N_{2}-124416 \zeta_{4} C_{2}^{4} d_{(1) 44} d_{(3) 44} d_{(4) 44} N_{2} \\
& -552960 \zeta_{5} C_{2}^{4} d_{(1) 44} d_{(3) 44} d_{(4) 44} N_{2}+718848 C_{2}^{4} d_{(1) 44} d_{(3) 44} d_{(4) 44} N_{2} \\
& +14976 \zeta_{3} C_{3}^{4} C_{4}^{4} d_{(1) 44} d_{(2) 44} N_{3} N_{4}+5184 \zeta_{4} C_{3}^{4} C_{4}^{4} d_{(1) 44} d_{(2) 44} N_{3} N_{4}
\end{aligned}
$$




$$
\begin{aligned}
& -46080 \zeta_{5} C_{3}^{4} C_{4}^{4} d_{(1) 44} d_{(2) 44} N_{3} N_{4}+29952 C_{3}^{4} C_{4}^{4} d_{(1) 44} d_{(2) 44} N_{3} N_{4} \\
& -27648 \zeta_{3} C_{3}^{4} d_{(1) 44} d_{(2) 44} d_{(4) 44} N_{3}-124416 \zeta_{4} C_{3}^{4} d_{(1) 44} d_{(2) 44} d_{(4) 44} N_{3} \\
& -552960 \zeta_{5} C_{3}^{4} d_{(1) 44} d_{(2) 44} d_{(4) 44} N_{3}+718848 C_{3}^{4} d_{(1) 44} d_{(2) 44} d_{(4) 44} N_{3} \\
& -27648 \zeta_{3} C_{4}^{4} d_{(1) 44} d_{(2) 44} d_{(3) 44} N_{4}-124416 \zeta_{4} C_{4}^{4} d_{(1) 44} d_{(2) 44} d_{(3) 44} N_{4} \\
& -552960 \zeta_{5} C_{4}^{4} d_{(1) 44} d_{(2) 44} d_{(3) 44} N_{4}+718848 C_{4}^{4} d_{(1) 44} d_{(2) 44} d_{(3) 44} N_{4} \\
& +4644864 \zeta_{3} d_{(1) 44} d_{(2) 44} d_{(3) 44} d_{(4) 44}+2985984 \zeta_{4} d_{(1) 44} d_{(2) 44} d_{(3) 44} d_{(4) 44} \\
& \left.-6635520 \zeta_{5} d_{(1) 44} d_{(2) 44} d_{(3) 44} d_{(4) 44}\right] \frac{g^{8}}{23887872 N_{1} N_{2} N_{3} N_{4}}+O\left(g^{10}\right)
\end{aligned}
$$

and

$$
\begin{aligned}
& \gamma_{m}(g)=-C_{1} C_{2} C_{3} C_{4} \frac{g^{2}}{2}+C_{1}^{2} C_{2}^{2} C_{3}^{2} C_{4}^{2} \frac{g^{4}}{96}+C_{1}^{3} C_{2}^{3} C_{3}^{3} C_{4}^{3}\left[3024 \zeta_{3}-43537\right] \frac{g^{6}}{221184} \\
& +\left[-619077 \zeta_{3} C_{1}^{4} C_{2}^{4} C_{3}^{4} C_{4}^{4} N_{1} N_{2} N_{3} N_{4}-91800 \zeta_{4} C_{1}^{4} C_{2}^{4} C_{3}^{4} C_{4}^{4} N_{1} N_{2} N_{3} N_{4}\right. \\
& -177630 \zeta_{5} C_{1}^{4} C_{2}^{4} C_{3}^{4} C_{4}^{4} N_{1} N_{2} N_{3} N_{4}+2070250 C_{1}^{4} C_{2}^{4} C_{3}^{4} C_{4}^{4} N_{1} N_{2} N_{3} N_{4} \\
& +14544 \zeta_{3} C_{1}^{4} C_{2}^{4} C_{3}^{4} d_{(4) 44} N_{1} N_{2} N_{3}-1296 \zeta_{4} C_{1}^{4} C_{2}^{4} C_{3}^{4} d_{(4) 44} N_{1} N_{2} N_{3} \\
& -39600 \zeta_{5} C_{1}^{4} C_{2}^{4} C_{3}^{4} d_{(4) 44} N_{1} N_{2} N_{3}+34128 C_{1}^{4} C_{2}^{4} C_{3}^{4} d_{(4) 44} N_{1} N_{2} N_{3} \\
& +14544 \zeta_{3} C_{1}^{4} C_{2}^{4} C_{4}^{4} d_{(3) 44} N_{1} N_{2} N_{4}-1296 \zeta_{4} C_{1}^{4} C_{2}^{4} C_{4}^{4} d_{(3) 44} N_{1} N_{2} N_{4} \\
& -39600 \zeta_{5} C_{1}^{4} C_{2}^{4} C_{4}^{4} d_{(3) 44} N_{1} N_{2} N_{4}+34128 C_{1}^{4} C_{2}^{4} C_{4}^{4} d_{(3) 44} N_{1} N_{2} N_{4} \\
& +231552 \zeta_{3} C_{1}^{4} C_{2}^{4} d_{(3) 44} d_{(4) 44} N_{1} N_{2}+31104 \zeta_{4} C_{1}^{4} C_{2}^{4} d_{(3) 44} d_{(4) 44} N_{1} N_{2} \\
& -293760 \zeta_{5} C_{1}^{4} C_{2}^{4} d_{(3) 44} d_{(4) 44} N_{1} N_{2}+273024 C_{1}^{4} C_{2}^{4} d_{(3) 44} d_{(4) 44} N_{1} N_{2} \\
& +14544 \zeta_{3} C_{1}^{4} C_{3}^{4} C_{4}^{4} d_{(2) 44} N_{1} N_{3} N_{4}-1296 \zeta_{4} C_{1}^{4} C_{3}^{4} C_{4}^{4} d_{(2) 44} N_{1} N_{3} N_{4} \\
& -39600 \zeta_{5} C_{1}^{4} C_{3}^{4} C_{4}^{4} d_{(2) 44} N_{1} N_{3} N_{4}+34128 C_{1}^{4} C_{3}^{4} C_{4}^{4} d_{(2) 44} N_{1} N_{3} N_{4} \\
& +231552 \zeta_{3} C_{1}^{4} C_{3}^{4} d_{(2) 44} d_{(4) 44} N_{1} N_{3}+31104 \zeta_{4} C_{1}^{4} C_{3}^{4} d_{(2) 44} d_{(4) 44} N_{1} N_{3} \\
& -293760 \zeta_{5} C_{1}^{4} C_{3}^{4} d_{(2) 44} d_{(4) 44} N_{1} N_{3}+273024 C_{1}^{4} C_{3}^{4} d_{(2) 44} d_{(4) 44} N_{1} N_{3} \\
& +231552 \zeta_{3} C_{1}^{4} C_{4}^{4} d_{(2) 44} d_{(3) 44} N_{1} N_{4}+31104 \zeta_{4} C_{1}^{4} C_{4}^{4} d_{(2) 44} d_{(3) 44} N_{1} N_{4} \\
& -293760 \zeta_{5} C_{1}^{4} C_{4}^{4} d_{(2) 44} d_{(3) 44} N_{1} N_{4}+273024 C_{1}^{4} C_{4}^{4} d_{(2) 44} d_{(3) 44} N_{1} N_{4} \\
& +1410048 \zeta_{3} C_{1}^{4} d_{(2) 44} d_{(3) 44} d_{(4) 44} N_{1}-746496 \zeta_{4} C_{1}^{4} d_{(2) 44} d_{(3) 44} d_{(4) 44} N_{1} \\
& -7879680 \zeta_{5} C_{1}^{4} d_{(2) 44} d_{(3) 44} d_{(4) 44} N_{1}+6552576 C_{1}^{4} d_{(2) 44} d_{(3) 44} d_{(4) 44} N_{1} \\
& +14544 \zeta_{3} C_{2}^{4} C_{3}^{4} C_{4}^{4} d_{(1) 44} N_{2} N_{3} N_{4}-1296 \zeta_{4} C_{2}^{4} C_{3}^{4} C_{4}^{4} d_{(1) 44} N_{2} N_{3} N_{4} \\
& -39600 \zeta_{5} C_{2}^{4} C_{3}^{4} C_{4}^{4} d_{(1) 44} N_{2} N_{3} N_{4}+34128 C_{2}^{4} C_{3}^{4} C_{4}^{4} d_{(1) 44} N_{2} N_{3} N_{4} \\
& +231552 \zeta_{3} C_{2}^{4} C_{3}^{4} d_{(1) 44} d_{(4) 44} N_{2} N_{3}+31104 \zeta_{4} C_{2}^{4} C_{3}^{4} d_{(1) 44} d_{(4) 44} N_{2} N_{3} \\
& -293760 \zeta_{5} C_{2}^{4} C_{3}^{4} d_{(1) 44} d_{(4) 44} N_{2} N_{3}+273024 C_{2}^{4} C_{3}^{4} d_{(1) 44} d_{(4) 44} N_{2} N_{3} \\
& +231552 \zeta_{3} C_{2}^{4} C_{4}^{4} d_{(1) 44} d_{(3) 44} N_{2} N_{4}+31104 \zeta_{4} C_{2}^{4} C_{4}^{4} d_{(1) 44} d_{(3) 44} N_{2} N_{4} \\
& -293760 \zeta_{5} C_{2}^{4} C_{4}^{4} d_{(1) 44} d_{(3) 44} N_{2} N_{4}+273024 C_{2}^{4} C_{4}^{4} d_{(1) 44} d_{(3) 44} N_{2} N_{4} \\
& +1410048 \zeta_{3} C_{2}^{4} d_{(1) 44} d_{(3) 44} d_{(4) 44} N_{2}-746496 \zeta_{4} C_{2}^{4} d_{(1) 44} d_{(3) 44} d_{(4) 44} N_{2}
\end{aligned}
$$




$$
\begin{aligned}
& -7879680 \zeta_{5} C_{2}^{4} d_{(1) 44} d_{(3) 44} d_{(4) 44} N_{2}+6552576 C_{2}^{4} d_{(1) 44} d_{(3) 44} d_{(4) 44} N_{2} \\
& +231552 \zeta_{3} C_{3}^{4} C_{4}^{4} d_{(1) 44} d_{(2) 44} N_{3} N_{4}+31104 \zeta_{4} C_{3}^{4} C_{4}^{4} d_{(1) 44} d_{(2) 44} N_{3} N_{4} \\
& -293760 \zeta_{5} C_{3}^{4} C_{4}^{4} d_{(1) 44} d_{(2) 44} N_{3} N_{4}+273024 C_{3}^{4} C_{4}^{4} d_{(1) 44} d_{(2) 44} N_{3} N_{4} \\
& +1410048 \zeta_{3} C_{3}^{4} d_{(1) 44} d_{(2) 44} d_{(4) 44} N_{3}-746496 \zeta_{4} C_{3}^{4} d_{(1) 44} d_{(2) 44} d_{(4) 44} N_{3} \\
& -7879680 \zeta_{5} C_{3}^{4} d_{(1) 44} d_{(2) 44} d_{(4) 44} N_{3}+6552576 C_{3}^{4} d_{(1) 44} d_{(2) 44} d_{(4) 44} N_{3} \\
& +1410048 \zeta_{3} C_{4}^{4} d_{(1) 44} d_{(2) 44} d_{(3) 44} N_{4}-746496 \zeta_{4} C_{4}^{4} d_{(1) 44} d_{(2) 44} d_{(3) 44} N_{4} \\
& -7879680 \zeta_{5} C_{4}^{4} d_{(1) 44} d_{(2) 44} d_{(3) 44} N_{4}+6552576 C_{4}^{4} d_{(1) 44} d_{(2) 44} d_{(3) 44} N_{4} \\
& +49766400 \zeta_{3} d_{(1) 44} d_{(2) 44} d_{(3) 44} d_{(4) 44}+17915904 \zeta_{4} d_{(1) 44} d_{(2) 44} d_{(3) 44} d_{(4) 44} \\
& \left.+9953280 \zeta_{5} d_{(1) 44} d_{(2) 44} d_{(3) 44} d_{(4) 44}\right] \frac{g^{8}}{23887872 N_{1} N_{2} N_{3} N_{4}}+O\left(g^{10}\right) .
\end{aligned}
$$

Finally, the $\beta$ function is

$$
\begin{aligned}
& \beta(g)=-3 C_{1} C_{2} C_{3} C_{4} \frac{g^{3}}{32}-467 C_{1}^{2} C_{2}^{2} C_{3}^{2} C_{4}^{2} \frac{g^{5}}{18432} \\
& +\left[48 \zeta_{3} C_{1}^{4} C_{2}^{4} C_{3}^{4} C_{4}^{4} N_{1} N_{2} N_{3} N_{4}-125981 C_{1}^{4} C_{2}^{4} C_{3}^{4} C_{4}^{4} N_{1} N_{2} N_{3} N_{4}\right. \\
& -1152 \zeta_{3} C_{1}^{4} C_{2}^{4} C_{3}^{4} d_{(4) 44} N_{1} N_{2} N_{3}+3456 C_{1}^{4} C_{2}^{4} C_{3}^{4} d_{(4) 44} N_{1} N_{2} N_{3} \\
& -1152 \zeta_{3} C_{1}^{4} C_{2}^{4} C_{4}^{4} d_{(3) 44} N_{1} N_{2} N_{4}+3456 C_{1}^{4} C_{2}^{4} C_{4}^{4} d_{(3) 44} N_{1} N_{2} N_{4} \\
& +27648 \zeta_{3} C_{1}^{4} C_{2}^{4} d_{(3) 44} d_{(4) 44} N_{1} N_{2}+27648 C_{1}^{4} C_{2}^{4} d_{(3) 44} d_{(4) 44} N_{1} N_{2} \\
& -1152 \zeta_{3} C_{1}^{4} C_{3}^{4} C_{4}^{4} d_{(2) 44} N_{1} N_{3} N_{4}+3456 C_{1}^{4} C_{3}^{4} C_{4}^{4} d_{(2) 44} N_{1} N_{3} N_{4} \\
& +27648 \zeta_{3} C_{1}^{4} C_{3}^{4} d_{(2) 44} d_{(4) 44} N_{1} N_{3}+27648 C_{1}^{4} C_{3}^{4} d_{(2) 44} d_{(4) 44} N_{1} N_{3} \\
& +27648 \zeta_{3} C_{1}^{4} C_{4}^{4} d_{(2) 44} d_{(3) 44} N_{1} N_{4}+27648 C_{1}^{4} C_{4}^{4} d_{(2) 44} d_{(3) 44} N_{1} N_{4} \\
& -663552 \zeta_{3} C_{1}^{4} d_{(2) 44} d_{(3) 44} d_{(4) 44} N_{1}+663552 C_{1}^{4} d_{(2) 44} d_{(3) 44} d_{(4) 44} N_{1} \\
& -1152 \zeta_{3} C_{2}^{4} C_{3}^{4} C_{4}^{4} d_{(1) 44} N_{2} N_{3} N_{4}+3456 C_{2}^{4} C_{3}^{4} C_{4}^{4} d_{(1) 44} N_{2} N_{3} N_{4} \\
& +27648 \zeta_{3} C_{2}^{4} C_{3}^{4} d_{(1) 44} d_{(4) 44} N_{2} N_{3}+27648 C_{2}^{4} C_{3}^{4} d_{(1) 44} d_{(4) 44} N_{2} N_{3} \\
& +27648 \zeta_{3} C_{2}^{4} C_{4}^{4} d_{(1) 44} d_{(3) 44} N_{2} N_{4}+27648 C_{2}^{4} C_{4}^{4} d_{(1) 44} d_{(3) 44} N_{2} N_{4} \\
& -663552 \zeta_{3} C_{2}^{4} d_{(1) 44} d_{(3) 44} d_{(4) 44} N_{2}+663552 C_{2}^{4} d_{(1) 44} d_{(3) 44} d_{(4) 44} N_{2} \\
& +27648 \zeta_{3} C_{3}^{4} C_{4}^{4} d_{(1) 44} d_{(2) 44} N_{3} N_{4}+27648 C_{3}^{4} C_{4}^{4} d_{(1) 44} d_{(2) 44} N_{3} N_{4} \\
& -663552 \zeta_{3} C_{3}^{4} d_{(1) 44} d_{(2) 44} d_{(4) 44} N_{3}+663552 C_{3}^{4} d_{(1) 44} d_{(2) 44} d_{(4) 44} N_{3} \\
& -663552 \zeta_{3} C_{4}^{4} d_{(1) 44} d_{(2) 44} d_{(3) 44} N_{4}+663552 C_{4}^{4} d_{(1) 44} d_{(2) 44} d_{(3) 44} N_{4} \\
& \left.+15925248 \zeta_{3} d_{(1) 44} d_{(2) 44} d_{(3) 44} d_{(4) 44}\right] \frac{g^{7}}{10616832 C_{1} C_{2} C_{3} C_{4} N_{1} N_{2} N_{3} N_{4}} \\
& +\left[394304 \zeta_{3} C_{1}^{4} C_{2}^{4} C_{3}^{4} C_{4}^{4} N_{1} N_{2} N_{3} N_{4}-15552 C_{1}^{4} C_{2}^{4} C_{3}^{4} C_{4}^{4} N_{1} N_{2} N_{3} N_{4} \zeta_{4}\right. \\
& +325120 C_{1}^{4} C_{2}^{4} C_{3}^{4} C_{4}^{4} N_{1} N_{2} N_{3} N_{4} \zeta_{5}-134800515 C_{1}^{4} C_{2}^{4} C_{3}^{4} C_{4}^{4} N_{1} N_{2} N_{3} N_{4} \\
& -5373696 \zeta_{3} C_{1}^{4} C_{2}^{4} C_{3}^{4} d_{(4) 44} N_{1} N_{2} N_{3}+373248 \zeta_{4} C_{1}^{4} C_{2}^{4} C_{3}^{4} d_{(4) 44} N_{1} N_{2} N_{3}
\end{aligned}
$$




$$
\begin{aligned}
& +4592640 \zeta_{5} C_{1}^{4} C_{2}^{4} C_{3}^{4} d_{(4) 44} N_{1} N_{2} N_{3}+3780864 C_{1}^{4} C_{2}^{4} C_{3}^{4} d_{(4) 44} N_{1} N_{2} N_{3} \\
& -5373696 \zeta_{3} C_{1}^{4} C_{2}^{4} C_{4}^{4} d_{(3) 44} N_{1} N_{2} N_{4}+373248 \zeta_{4} C_{1}^{4} C_{2}^{4} C_{4}^{4} d_{(3) 44} N_{1} N_{2} N_{4} \\
& +4592640 \zeta_{5} C_{1}^{4} C_{2}^{4} C_{4}^{4} d_{(3) 44} N_{1} N_{2} N_{4}+3780864 C_{1}^{4} C_{2}^{4} C_{4}^{4} d_{(3) 44} N_{1} N_{2} N_{4} \\
& -37361664 \zeta_{3} C_{1}^{4} C_{2}^{4} d_{(3) 44} d_{(4) 44} N_{1} N_{2}-8957952 \zeta_{4} C_{1}^{4} C_{2}^{4} d_{(3) 44} d_{(4) 44} N_{1} N_{2} \\
& +79994880 \zeta_{5} C_{1}^{4} C_{2}^{4} d_{(3) 44} d_{(4) 44} N_{1} N_{2}+31574016 C_{1}^{4} C_{2}^{4} d_{(3) 44} d_{(4) 44} N_{1} N_{2} \\
& -5373696 \zeta_{3} C_{1}^{4} C_{3}^{4} C_{4}^{4} d_{(2) 44} N_{1} N_{3} N_{4}+373248 \zeta_{4} C_{1}^{4} C_{3}^{4} C_{4}^{4} d_{(2) 44} N_{1} N_{3} N_{4} \\
& +4592640 \zeta_{5} C_{1}^{4} C_{3}^{4} C_{4}^{4} d_{(2) 44} N_{1} N_{3} N_{4}+3780864 C_{1}^{4} C_{3}^{4} C_{4}^{4} d_{(2) 44} N_{1} N_{3} N_{4} \\
& -37361664 \zeta_{3} C_{1}^{4} C_{3}^{4} d_{(2) 44} d_{(4) 44} N_{1} N_{3}-8957952 \zeta_{4} C_{1}^{4} C_{3}^{4} d_{(2) 44} d_{(4) 44} N_{1} N_{3} \\
& +79994880 \zeta_{5} C_{1}^{4} C_{3}^{4} d_{(2) 44} d_{(4) 44} N_{1} N_{3}+31574016 C_{1}^{4} C_{3}^{4} d_{(2) 44} d_{(4) 44} N_{1} N_{3} \\
& -37361664 \zeta_{3} C_{1}^{4} C_{4}^{4} d_{(2) 44} d_{(3) 44} N_{1} N_{4}-8957952 \zeta_{4} C_{1}^{4} C_{4}^{4} d_{(2) 44} d_{(3) 44} N_{1} N_{4} \\
& +79994880 \zeta_{5} C_{1}^{4} C_{4}^{4} d_{(2) 44} d_{(3) 44} N_{1} N_{4}+31574016 C_{1}^{4} C_{4}^{4} d_{(2) 44} d_{(3) 44} N_{1} N_{4} \\
& -1895546880 \zeta_{3} C_{1}^{4} d_{(2) 44} d_{(3) 44} d_{(4) 44} N_{1}+214990848 \zeta_{4} C_{1}^{4} d_{(2) 44} d_{(3) 44} d_{(4) 44} N_{1} \\
& +1159004160 \zeta_{5} C_{1}^{4} d_{(2) 44} d_{(3) 44} d_{(4) 44} N_{1}+630374400 C_{1}^{4} d_{(2) 44} d_{(3) 44} d_{(4) 44} N_{1} \\
& -5373696 \zeta_{3} C_{2}^{4} C_{3}^{4} C_{4}^{4} d_{(1) 44} N_{2} N_{3} N_{4}+373248 \zeta_{4} C_{2}^{4} C_{3}^{4} C_{4}^{4} d_{(1) 44} N_{2} N_{3} N_{4} \\
& +4592640 \zeta_{5} C_{2}^{4} C_{3}^{4} C_{4}^{4} d_{(1) 44} N_{2} N_{3} N_{4}+3780864 C_{2}^{4} C_{3}^{4} C_{4}^{4} d_{(1) 44} N_{2} N_{3} N_{4} \\
& -37361664 \zeta_{3} C_{2}^{4} C_{3}^{4} d_{(1) 44} d_{(4) 44} N_{2} N_{3}-8957952 \zeta_{4} C_{2}^{4} C_{3}^{4} d_{(1) 44} d_{(4) 44} N_{2} N_{3} \\
& +79994880 \zeta_{5} C_{2}^{4} C_{3}^{4} d_{(1) 44} d_{(4) 44} N_{2} N_{3}+31574016 C_{2}^{4} C_{3}^{4} d_{(1) 44} d_{(4) 44} N_{2} N_{3} \\
& -37361664 \zeta_{3} C_{2}^{4} C_{4}^{4} d_{(1) 44} d_{(3) 44} N_{2} N_{4}-8957952 \zeta_{4} C_{2}^{4} C_{4}^{4} d_{(1) 44} d_{(3) 44} N_{2} N_{4} \\
& +79994880 \zeta_{5} C_{2}^{4} C_{4}^{4} d_{(1) 44} d_{(3) 44} N_{2} N_{4}+31574016 C_{2}^{4} C_{4}^{4} d_{(1) 44} d_{(3) 44} N_{2} N_{4} \\
& -1895546880 \zeta_{3} C_{2}^{4} d_{(1) 44} d_{(3) 44} d_{(4) 44} N_{2}+214990848 \zeta_{4} C_{2}^{4} d_{(1) 44} d_{(3) 44} d_{(4) 44} N_{2} \\
& +1159004160 \zeta_{5} C_{2}^{4} d_{(1) 44} d_{(3) 44} d_{(4) 44} N_{2}+630374400 C_{2}^{4} d_{(1) 44} d_{(3) 44} d_{(4) 44} N_{2} \\
& -37361664 \zeta_{3} C_{3}^{4} C_{4}^{4} d_{(1) 44} d_{(2) 44} N_{3} N_{4}-8957952 \zeta_{4} C_{3}^{4} C_{4}^{4} d_{(1) 44} d_{(2) 44} N_{3} N_{4} \\
& +79994880 \zeta_{5} C_{3}^{4} C_{4}^{4} d_{(1) 44} d_{(2) 44} N_{3} N_{4}+31574016 C_{3}^{4} C_{4}^{4} d_{(1) 44} d_{(2) 44} N_{3} N_{4} \\
& -1895546880 \zeta_{3} C_{3}^{4} d_{(1) 44} d_{(2) 44} d_{(4) 44} N_{3}+214990848 \zeta_{4} C_{3}^{4} d_{(1) 44} d_{(2) 44} d_{(4) 44} N_{3} \\
& +1159004160 \zeta_{5} C_{3}^{4} d_{(1) 44} d_{(2) 44} d_{(4) 44} N_{3}+630374400 C_{3}^{4} d_{(1) 44} d_{(2) 44} d_{(4) 44} N_{3} \\
& -1895546880 \zeta_{3} C_{4}^{4} d_{(1) 44} d_{(2) 44} d_{(3) 44} N_{4}+214990848 \zeta_{4} C_{4}^{4} d_{(1) 44} d_{(2) 44} d_{(3) 44} N_{4} \\
& +1159004160 \zeta_{5} C_{4}^{4} d_{(1) 44} d_{(2) 44} d_{(3) 44} N_{4}+630374400 C_{4}^{4} d_{(1) 44} d_{(2) 44} d_{(3) 44} N_{4} \\
& -3301834752 \zeta_{3} d_{(1) 44} d_{(2) 44} d_{(3) 44} d_{(4) 44}-5159780352 \zeta_{4} d_{(1) 44} d_{(2) 44} d_{(3) 44} d_{(4) 44} \\
& \left.+24418713600 \zeta_{5} d_{(1) 44} d_{(2) 44} d_{(3) 44} d_{(4) 44}\right] \frac{g^{9}}{18345885696 N_{1} N_{2} N_{3} N_{4}} \text {. } \\
& +O\left(g^{11}\right)
\end{aligned}
$$


[1] D. J. Gross and F. J. Wilczek, Phys. Rev. Lett. 30, 1343 (1973).

[2] H. D. Politzer, Phys. Rev. Lett. 30, 1346 (1973).

[3] E. Ma, Prog. Theor. Phys. 54, 1828 (1975).

[4] A. J. McKane, D. J. Wallace, and R. K. P. Zia, Phys. Lett. 65B, 171 (1976).

[5] A. J. McKane, J. Phys. G 3, 1165 (1977).

[6] B. M. McCoy and T. T. Wu, Phys. Rev. D 12, 578 (1975).

[7] M. C. Bergère and C. Gilain, J. Math. Phys. (N.Y.) 19, 1495 (1978).

[8] R. Hong Tuan, Phys. Lett. B 393, 84 (1997).

[9] C. M. Fortuin and P. W. Kasteyn, Physica (Amsterdam) 57, 536 (1972).

[10] R. K. P. Zia and D. J. Wallace, J. Phys. A 8, 1495 (1975).

[11] M. E. Fisher, Phys. Rev. Lett. 40, 1610 (1978).

[12] Z. Bern, J. J. M. Carrasco, and H. Johansson, Phys. Rev. D 78, 085011 (2008).

[13] Z. Bern, T. Dennen, Y.-T. Huang, and M. Kiermaier, Phys. Rev. D 82, 065003 (2010).

[14] H. Kawai, D. C. Lewellen, and S. H. Tye, Nucl. Phys. B269, 1 (1986).

[15] F. Cachazi, S. He, and E. Y. Yuan, J. High Energy Phys. 07 (2014) 033.

[16] A. Anastasiou, L. Borsten, M. J. Duff, L. J. Hughes, and S. Nagy, Phys. Rev. Lett. 113, 231606 (2014).

[17] A. Parkes, Phys. Lett. B 286, 265 (1992).

[18] R. Monteiro, D. O'Connell, and C. D. White, J. High Energy Phys. 12 (2014) 056.

[19] A. Luna, R. Monteiro, D. O'Connell, and C. D. White, Phys. Lett. B 750, 272 (2015).

[20] W. D. Goldberger, S. G. Prabhu, and J. O. Thompson, Phys. Rev. D 96, 065009 (2017).
[21] N. Bahjat-Abbas, R. Stark-Muchão, and C. D. White, Phys. Lett. B 788, 274 (2019).

[22] T. van Ritbergen, J. A. M. Vermaseren, and S. A. Larin, Phys. Lett. B 400, 379 (1997).

[23] T. van Ritbergen, A. N. Schellekens, and J. A. M. Vermaseren, Int. J. Mod. Phys. A 14, 41 (1999).

[24] J. A. Gracey, Phys. Rev. D 92, 025012 (2015).

[25] A. J. Macfarlane and G. Woo, Nucl. Phys. B77, 91 (1974); B86, 548(E) (1975).

[26] O. F. de Alcantara Bonfim, J.E. Kirkham, and A. J. McKane, J. Phys. A 13, L247 (1980); 13, 3785(E) (1980).

[27] O.F. de Alcantara Bonfim, J.E. Kirkham, and A. J. McKane, J. Phys. A 14, 2391 (1981).

[28] S. Laporta, Int. J. Mod. Phys. A 15, 5087 (2000).

[29] P. A. Baikov and K. G. Chetyrkin, Nucl. Phys. B837, 186 (2010).

[30] O. V. Tarasov, Phys. Rev. D 54, 6479 (1996).

[31] O. V. Tarasov, Nucl. Phys. B502, 455 (1997).

[32] C. Studerus, Comput. Phys. Commun. 181, 1293 (2010).

[33] A. von Manteuffel and C. Studerus, arXiv:1201.4330.

[34] J. A. M. Vermaseren, arXiv:math-ph/0010025.

[35] M. Tentyukov and J. A. M. Vermaseren, Comput. Phys. Commun. 181, 1419 (2010).

[36] P. Nogueira, J. Comput. Phys. 105, 279 (1993).

[37] S. A. Larin and J. A. M. Vermaseren, Phys. Lett. B 303, 334 (1993).

[38] T. Banks and A. Zaks, Nucl. Phys. B196, 189 (1982).

[39] K. G. Wilson and M. E. Fisher, Phys. Rev. Lett. 28, 240 (1972).

[40] K. G. Wilson, Phys. Rep. 12, 75 (1974).

[41] S. R. Coleman and D. J. Gross, Phys. Rev. Lett. 31, 851 (1973). 